Z Herz- Thorax- Gefäßchir 2019 · 33:1

https://doi.org/10.1007/s00398-018-0289-8

(C) Springer Medizin Verlag $\mathrm{GmbH}$, ein Teil von Springer Nature 2018

CrossMark

\author{
A. Krian' $\cdot$ M. Heinemann ${ }^{2}$ \\ 'Deutsche Gesellschaft für Thorax-, Herz- und Gefäßchirurgie, Berlin, Deutschland \\ ${ }^{2}$ Klinik und Poliklinik für Herz-, Thorax- und Gefäßchirurgie, Universitätsmedizin Mainz, Mainz, \\ Deutschland
}

\title{
Die Rubrik „Historische Profile“
}

Liebe Leserinnen und Leser,

in diesem Heft erscheint erstmals die neue Rubrik: „Historische Profile“. In ihr stellt Prof. Herbert Ulmer, Emeritus der Abteilung Pädiatrische Kardiologie am Universitätsklinikum Heidelberg, Persönlichkeiten vor, die für die Entwicklung von Kinderherzchirurgie und Kinderkardiologie maßgeblich waren. Wie kaum bei anderen Fächern ist die enge Verzahnung dieser beiden Disziplinen eine natürlich und historisch gewachsene Selbstverständlichkeit. Sie dient zudem als Beispiel, wie und dass man aus der Geschichte lernen kann und sollte. Man denke nur an das heute so viel gepriesene, weil plötzlich anderweitig neu entdeckte „Heart-Team“.

\section{》) Innehalten und sich zurückbesinnen auf die Vergangenheit und die Geschichte, die uns geprägt hat}

In unserer schnelllebigen Zeit tut es Not, gelegentlich innezuhalten und sich zurückzubesinnen auf die Vergangenheit, auf die Geschichte, die uns alle geprägt hat. In der Medizin tragen Techniken und Prozeduren gerne den Namen der/des (vermeintlich) Erstbeschreibenden. Man denke nur an Blalock und Taussig, Senning und Mustard, Rashkind, Norwood, Fontan - alles Namen im täglichen Gebrauch. Aber wissen wir auch, welche Menschen sich dahinter verbergen, unter welchen Umständen diese Pionierleistungen entstanden? Und dann gibt es noch die, deren Namen nicht zum Synonym von Eingriffen geworden sind, ohne die aber die moderne Kinderkardiolo- gie und -herzchirurgie undenkbar wären: z. B. Maude Abbott, Alexander Nadas, Stella und Richard Van Praagh, Aldo Castaneda.

Begeben wir uns mit Herbert Ulmer auf eine Reise in die jüngere Vergangenheit. Sie wird mit Sicherheit für eine spannende Mischung aus Ver- und Bewunderung sorgen.

\section{Prof. Dr. Arno Krian}

Chronist der Deutschen Gesellschaft für Thorax-, Herz- und Gefäßchirurgie

Prof. Dr. Markus Heinemann Rubrikherausgeber der Zeitschrift für Herz-, Thorax- und Gefäßchirurgie

\section{Korrespondenzadresse}

\section{Prof. Dr. M. Heinemann}

Klinik und Poliklinik für Herz-, Thorax- und Gefäßchirurgie, Universitätsmedizin Mainz Langenbeckstr. 1, 55131 Mainz, Deutschland mheinema@uni-mainz.de

Interessenkonflikt. A. Krian und M. Heinemann geben an, dass kein Interessenkonflikt besteht. 